\title{
Ecos históricos de la enseñanza del inglés en la escuela primaria, Costa Rica PPEIEP: 1979- $1987^{1}$
}

\section{(Historical Echoes of Teaching English in Primary School in Costa Rica PPEIEP: 1979-1987)}

\author{
Nandayure Valenzuela Arce ${ }^{2}$ \\ Universidad Nacional, Costa Rica \\ Gustavo Álvarez Martínez ${ }^{3}$ \\ Universidad Nacional, Costa Rica
}

\begin{abstract}
resumen
Este es uno de un grupo de estudios que presentan una visión integral del quehacer pionero de la Escuela de Literatura y Ciencias del Lenguaje (ELCL) de la Universidad Nacional de Costa Rica en cuanto al desarrollo de la enseñanza comunicativa del inglés a población infantil en nuestro país. Es la reconstrucción histórica del primer proyecto de extensión, en este campo, de la ELCL, denominado Proyecto Piloto de la Enseñanza del Inglés en la Escuela Primaria Pública [1979-1987]. Se informa acerca de las dinámicas del proyecto y sus efectos en la comunidad de docentes de inglés y el estudiantado de primaria en la educación pública.
\end{abstract}

1 Recibido: 10 de mayo de 2013; aceptado: 11 de septiembre de 2013. Estudio desarrollado durante una primera etapa del Proyecto de Extensión No. 0556-11 (2009-2014), Programa de Capacitación para Maestros de Inglés (PROCAMI), de la Universidad Nacional, por Nandayure Valenzuela Arce (responsable) y Gustavo Álvarez Martínez (participante).

2 Escuela de Literatura y Ciencias del Lenguaje. Correo electrónico: nandayureva@ gmail.com

3 Escuela de Literatura y Ciencias del Lenguaje. Correo electrónico: lgtavo@yahoo.com 


\begin{abstract}
This is one of several studies which present a comprehensive overview of the pioneering efforts of the Escuela de Literatura y Ciencias del Lenguaje (ELCL) at the Universidad Nacional, Costa Rica, in the development of communicative English teaching to elementary school children. Thus, our first effort is to recover the historical memory of the first outreach project developed by the ELCL for that purpose, known as the Pilot Project for the Teaching of English in Primary Public Schools [1979-1987] (Proyecto Piloto de la Enseñanza del Inglés en la Escuela Primaria Pública). Information is provided on the dynamics of the project and its impact on English teachers and students at the public elementary school level.
\end{abstract}

Palabras clave: enseñanza comunicativa de idiomas, enseñanza del inglés para educación primaria

Keywords: communicative language teaching, teaching English as a foreign language in primary school

\title{
Panorama general
}

Este estudio procura sistematizar los esfuerzos realizados por algunas docentes de la Escuela de Literatura y Ciencias del Lenguaje (ELCL) de la Universidad Nacional de Costa Rica (UNA) entre 1979 y 1987, que propone una opción innovadora y pertinente a la enseñanza del inglés a niños en el país, denominada Proyecto Piloto de la Enseñanza del Inglés en la Escuela Primaria Pública (PPEIEP). El diseño de investigación utilizado para estudiar los antecedentes del proyecto es narrativo autobiográfico, y para triangular la información realizamos procesos de análisis multidisciplinarios. Empleamos el esquema estructura-problema-solución, por lo que se narra el contexto, caracterizaciones, acciones, problemas y resoluciones ${ }^{4}$. La pregunta que orienta este estudio es: ¿Cuáles fueron las dinámicas pioneras de la ELCL para responder a la urgente necesidad de cimentar el bilingüismo en las escuelas públicas de Costa Rica entre

4 Roberto Hernández Sampieri, Carlos Fernández Collado y Pilar Baptista Lucio, Metodología de la investigación. 4ta. ed. (México, D. F.: McGraw-Hill. 2006) 705. 
1979 y 1987? Algunos de los instrumentos de recolección de datos utilizados son: 1) artefactos: memorias de la ELCL; documentos institucionales (tales como el estatuto orgánico de la UNA); informes finales del proyecto; producción de los extensionistas (ponencias, artículos, libros, cuadernos, fotos y otros); documentos históricos de la ELCL que revelan el quehacer del primer proyecto de extensión; y 2) entrevistas abiertas a las extensionistas pioneras involucradas en el PPEIEP.

\section{Plan Piloto PPEIEP: iniciativa pionera}

Antes de la década de 1970, en Costa Rica - como en otros países del mundo- el inglés en el sistema de educación pública se impartía solamente a nivel de secundaria y esta formación no se orientaba necesariamente a desarrollar la competencia comunicativa de los estudiantes. El aprendizaje de la lengua tenía más bien el propósito de posibilitar la comprensión de textos en inglés y consolidar el aprendizaje de la gramática de la lengua materna al compararla y contrastarla con la del inglés, y en última instancia, para enriquecer la cultura general del aprendiente ${ }^{5}$.

A mediados de 1970 se vislumbra la evolución de enfoques distintos para la enseñanza y aprendizaje del inglés, como resultado de los cambios que se venían gestando en la política económica a nivel mundial, y por ende en Costa Rica. La nueva dinámica financiera generó la apertura de las fronteras regionales y la flexibilización de políticas a nivel global y local, lo que puso en evidencia la necesidad de formar un recurso humano bilingüe, primordialmente en el idioma inglés 6 . Para atender esto, el Ministerio de Educación

5 Diane Larsen-Freeman, Techniques and Principles in Language Teaching (Oxford: Oxford University Press, 1986) 4.

6 Edwin Marín Arroyo, «El análisis de los programas de gobierno de la década de 1990, en relación con la enseñanza del Inglés en Costa Rica». San José, C.R.: Revista SEP-EDUCAR. Universidad Estatal a Distancia: Estudios de Posgrado, 2004, <http://www.uned.ac.cr/sep/info/index.htm>; consultado el 14 de marzo de 2010. 
Pública (MEP) contaba con la previa incorporación del inglés en el currículo de secundaria, el cual llevaba muchos años en ejecución. No obstante, no habilitaban a los estudiantes a comunicarse en el idioma extranjero, en vista de que el enfoque de enseñanza utilizado se centraba en desarrollar la habilidad de comprender textos. Por lo tanto, los graduados de colegios públicos no podían satisfacer la emergente necesidad nacional de recurso humano bilingüe. Así las cosas, dos condiciones limitaban la formación en lengua extranjera de la fuerza laboral nacional: 1) la utilización de metodología de enseñanza (enfoque gramatical y de traducción) que se implementaba en aquella época, la cual no tenía como fin el desarrollo de habilidades de comunicación oral y escrita, y 2) la exigua exposición de los discentes al idioma extranjero (dos lecciones de cuarenta minutos cada una, tres veces por semana).

Considerando este escenario, la ELCL aprovecha la oportunidad que se le presenta a finales de la década de 1970 de gestar un proyecto de enseñanza del inglés a niños, al plantearse un intercambio de colaboración interinstitucional entre la UNA y la Escuela Joaquín Lizano $^{7}$. Reuben y Vargas describen a continuación el proceso que dio lugar a esta circunstancia. Indican que desde mediados del decenio de 1970 existía en el Área de Inglés de la ELCL la preocupación por atender la necesidad nacional de desarrollar en los ciudadanos la habilidad de comunicarse en inglés desde su infancia, porque ello extendería el tiempo de exposición del estudiante a la lengua extranjera y se aprovecharía la flexibilidad cognitiva y emocional que tiene la población infantil para adquirir conocimiento nuevo. Sin embargo, no se habían dado los medios para gestar un proyecto con este fin,

7 Escuela construida como parte de la reforma educativa por la cual se encontraba Costa Rica en el decenio de 1880, impulsada por Mauro Fernández y Bernardo Soto. Edificio construido de ladrillo con cal y canto. Su nombre en honor al político de origen herediano, don Joaquín Lizano Gutiérrez quien fungió como Gobernador, Senador, Ministro de Hacienda y Presidente provisional. Declarada de interés histórico arquitectónico el 28 de mayo de 1992. Disponible en: <http://www. costaricaexplorerguide.com/php/atracciones2.php?idm=1\&atract=187>; consultado el 8 de julio de 2014. 
hasta que la solución a un problema institucional permitió establecer las condiciones requeridas. En 1978 la UNA enfrentaba un déficit en aulas para impartir cursos, en virtud de dos factores: 1. su acelerado desarrollo, el cual se había traducido en la multiplicación de carreras $\mathrm{y}$, consecuentemente, en la de su población estudiantil; y 2. el rezago en la construcción de nuevos espacios físicos, debido a restricciones presupuestarias. Alfio Piva Mesén, rector entonces de la UNA, atendió la situación estableciendo alianzas con otras instituciones, como medida apremiante para resolver el problema. Uno de estos pactos fue el que realizó con la Escuela Joaquín Lizano, aprovechando el vínculo que tenía con este centro educativo, en el cual estudiaba uno de sus hijos. Solicitó el apoyo de la Sra. Margarita Segnini, directora del centro educativo, proponiéndole que autorizara el préstamo de aulas de la Escuela Joaquín Lizano para que la UNA impartiera clases. La Sra. Segnini acepta, pero exhorta a que en reciprocidad la universidad imparta Inglés a los estudiantes de primer grado de la Escuela Joaquín Lizano y que la instrucción se ofrezca a los mismos pupilos de manera ininterrumpida, hasta que se gradúen en sexto grado. El señor rector consciente en lo solicitado por doña Margarita y deja en manos de la ELCL el cumplimiento de su petición ${ }^{8}$.

En 1978 la ELCL inaugura la enseñanza del inglés a niños en la educación pública mediante el diseño de un proyecto de extensión e investigación. La iniciativa tiene pertinencia al ser congruente con las áreas de interés de la ELCL y con su misión de promover la investigación del quehacer lingüístico y la producción de materiales que apoyen la enseñanza y el aprendizaje de lenguas ${ }^{9}$. Bajo este marco formal, entre 1979 y 1987 la ELCL emprendió de manera sistemática la tarea de generar mecanismos para que los costarricenses tuvieran acceso al aprendizaje del inglés desde una edad temprana.

8 Sonya Vargas y Ruth Reuben, Entrevista con Valenzuela y Álvarez. Campus Omar Dengo, Universidad Nacional. Heredia, Costa Rica. 30 de junio de 2014.

9 Escuela de Literatura y Ciencias del Lenguaje. «Misión y Visión». 〈http://www.literatura.una. ac.cr/>; consultado el 17 de diciembre de 2012. 
Esta iniciativa consistió en la formulación y desarrollo del PPEIEP, propuesto por las académicos Sonya Vargas Sáenz, Ruth Reuben Coronado, Claudina Quintana y Oscar Chavarría Aguilar - equipo de docentes universitarios de la ELCL- quienes indican que el proyecto responde a la necesidad «...de crear un modelo alternativo de enseñanza con métodos y programas originales [para la enseñanza del inglés a niños] que partan de nuestra realidad, ajustados a las necesidades inmediatas de los educandos y del país» ${ }^{10}$.

El PPEIEP fue concebido como un proyecto integrado de docencia, investigación y extensión, áreas que se ven reflejadas en los siguientes objetivos: 1. tomar en cuenta la realidad costarricense para la creación de materiales didácticos, lo que haría mucho más pertinente y efectivo el aprendizaje del inglés en nuestro contexto educativo $^{11} ; 2$. diseñar una oferta curricular para primaria y paralelamente ir probando su efectividad, de manera que la teoría ofreciera orientación a la práctica docente y esta a su vez enriqueciera el currículo en experimentación ${ }^{12} ;$ y 3 . que la experiencia de este plan piloto aportara información del dominio natural en el que se dan los procesos de enseñanza y aprendizaje de la lengua meta, para que esos datos nutrieran los currículos de los programas de la ELCL ${ }^{13}$. Con el fin de alcanzar estos objetivos se estableció un acuerdo con la Escuela Joaquín Lizano, institución ubicada en la provincia de Heredia, perteneciente al sistema de educación pública, cuyas autoridades contribuyeron a establecer las condiciones para el desarrollo exitoso del proyecto piloto en ese centro educativo. El PPEIEP se llevó a cabo en tres fases: 1. entre 1979 y 1982 se ejecuta la primera etapa del proyecto, durante la cual se imparten clases a los

10 Sonya Vargas, Ruth Reuben, Claudina Quintana y Oscar Chavarría, Propuesta de Extensión: Plan Piloto de Enseñanza del Inglés en la Escuela Primaria, 1979.

11 Vargas, Reuben, Quintana y Chavarría.

12 Ileana Villalobos Ellis, Entrevista con Valenzuela y Álvarez. Centro Educativo Yurusti, Santo Domingo de Heredia, Costa Rica, 12 de abril de 2011.

13 Memoria XX Aniversario de la Escuela de Literatura y Ciencias del Lenguaje 1974-1994. Heredia, Costa Rica: ELCL, 1994. 
estudiantes de primero, segundo y tercer grado; 2. entre 1983 y 1985 se implementa la segunda fase, atendiendo la enseñanza del inglés de los niños en cuarto, quinto y sexto grado; y 3. entre 1986 y 1987 se realiza la evaluación de la experiencia de práctica docente y de investigación-acción.

Durante las primeras dos fases señaladas — con una duración total de seis años (1979-1985) — se imparten lecciones de inglés a un promedio de cincuenta estudiantes, distribuidos en dos grupos de escolares, aunque se presentan períodos de fluctuación en el número de niños participantes por razones de índole presupuestaria. Hay etapas en las que las extensionistas trabajan impartiendo clase a tres grupos de estudiantes de primaria y otros en los que se reduce a tan solo un grupo.

Simultáneamente a este trabajo de formación, el equipo de extensionistas del PPEIEP llevó a cabo procesos de investigación-acción. Para impartir las clases diseñaron el currículo y los materiales didácticos originales y pertinentes al contexto educativo costarricense. Mediante la investigación, las extensionistas pilotearon la eficiencia de la metodología de enseñanza que utilizan para implementar el currículo de inglés en la Escuela Joaquín Lizano, así como la pertinencia de este currículo y los correspondientes materiales didácticos. El resultado de las investigaciones se utiliza para marcar la ruta a seguir, a fin de depurar todos los productos de enseñanza, poniendo de manifiesto las necesidades emergentes del estudiantado infantil. A partir de los resultados, el equipo de proyectistas realizaba las mejoras y nuevamente investigaba su impacto en la población meta, repitiéndose este proceso cíclico de creación, puesta en práctica e investigación durante los seis años en que se impartieron clases en la escuela ${ }^{14}$. Aún más, la investigación no solo tiene un gran impacto sobre el quehacer del proyecto in situ, sino que deja su huella a nivel nacional e internacional, pues los productos académicos innovadores que surgen de la experiencia se socializan a través de artículos,

14 Sonya Vargas, y Ruth Reuben, Plan Piloto de la Enseñanza del Inglés como Segunda Lengua: Parte I del Informe de 1979. Escuela de Literatura y Ciencias del Lenguaje, Universidad Nacional, 1979. 
ponencias, libros, y otros, para ir delineando el estado de la cuestión de la enseñanza del inglés a niños en Costa Rica. El equipo de trabajo del PPEIEP contó con ocho extensionistas que se consolidaron como tales a lo largo de casi una década en que el proyecto estuvo en operación. Estas proyectistas innovadoras del PPEIEP (1979-1987) son: Sonya Vargas Sáenz, Ruth Reuben Coronado, Claudina Quintana, Oscar Chavarría Aguilar, Ileana Villalobos Ellis, Lelia Villalobos Rodríguez, Ileana Saborío Pérez y Agnes Flores Gámez.

Las primeras dos extensionistas fungieron como coordinadoras del proyecto en diferentes períodos y permanecieron en él durante más tiempo que otros miembros del equipo de trabajo. Las otras integrantes formaron parte en diversos momentos, por lo que el PPEIEP contó con un recurso humano de tres extensionistas fijos, que eran sustituidos por otros compañeros con el paso del tiempo. Esta dinámica redundó en la formación de un total de ocho profesionales versados en extensión universitaria al finalizar el proyecto.

\section{Primera y segunda fase del Plan Piloto. Investigación y docencia (1979-1985)}

Este primer período de trabajo fue implementado por las profesoras Ruth Reuben y Sonya Vargas mediante la división de tareas: 1. el estudio de los procesos de enseñanza y aprendizaje del inglés; 2 . la elaboración de los programas e implementación de las lecciones; y 3. la documentación de los resultados de la aplicación de la teoría de enseñanza-aprendizaje utilizada en la población seleccionada de la Escuela Joaquín Lizano ${ }^{15}$. Las profesoras Vargas y Reuben estaban a cargo de impartir las lecciones de inglés, mientras que todos los miembros del equipo ${ }^{16}$ (incluidas las dos académicas antes mencionadas) tenían la responsabilidad de realizar investigación paralela. Para la investigación, se contó con los dos grupos de primer grado

15 Villalobos Ellis, Entrevista, 12 de abril de 2011.

16 Claudina Quintana, Oscar Chavarría Aguilar, Sonya Vargas Sáenz y Ruth Reuben Coronado. 
que iniciaron su instrucción primaria en la Escuela Joaquín Lizano en 1979. Los discentes recibían dos clases de inglés tres veces por semana, para un total de seis sesiones semanales. Cada grupo consistía de aproximadamente 25 estudiantes, incluidos algunos niños del aula diferenciada, constituida por estudiantes con necesidades educativas especiales que, según indica el Centro Nacional de Recursos para la Inclusión Educativa (CENAREC), del Ministerio de Educación Pública ${ }^{17}$ :

...es aquel estudiante que presenta condiciones de aprendizaje diferentes o dificultades en el aprendizaje mayores que el promedio de los alumnos, lo que le dificulta o impide acceder al currículo que le corresponde por su edad, de forma que requiere para compensar dichas diferencias, adecuaciones en una o varias áreas del currículo (...) adecuaciones de acceso (...) adecuaciones no significativas (...) adecuaciones significativas.

$\mathrm{Al}$ respecto, Vargas ${ }^{18}$ indica que había niños sordos a los que les impartían clase. Aunque al principio ellas (las extensionistas) se preocuparon un poco por este hecho, pronto su alarma se disipó porque la metodología de enseñanza que empleaban tenía tan motivados tanto a los estudiantes que no presentaban ningún tipo de necesidad especial, como a aquellos que sí las tenían, incluidos los estudiantes con discapacidad auditiva. Estos últimos avanzaron en su desarrollo de habilidades en inglés de manera sorprendente; su pronunciación en la lengua extranjera llegó a ser destacada y era muy activa su participación en las actividades de aprendizaje que se realizaban dentro y fuera de la clase.

Hasta ese momento, según Ileana Villalobos Ellis, la enseñanza de la lengua extranjera en el país se impartía a partir de textos impresos y auditivos que exponían al aprendiente a un

17 Centro Nacional de Recursos para la Inclusión Educativa del Ministerio de Educación Pública. Políticas, Normativa y Procedimientos para el Acceso a la Educación de los Estudiantes con Necesidades Educativas Especiales (San José, Costa Rica. 2005) 14-15.

18 Vargas y Reuben. Entrevista, 30 de junio de 2014. 
contexto cultural extranjero, muy distante de la idiosincrasia costarricense, por lo que urgía que el Plan Piloto adoptara estrategias para incluir contenidos que fueran familiares para la población infantil. Por ese motivo se planteó que la enseñanza del inglés a los estudiantes de la Escuela Joaquín Lizano se realizara incorporando contenidos de los programas oficiales de estudios sociales, matemáticas, ciencias y español del Ministerio de Educación Pública (MEP) ${ }^{19}$. A nivel de primaria, estas materias se centran en el contexto costarricense: geografía y cultura del país, procesos matemáticos básicos aplicados a la realidad cultural del estudiante, flora y fauna costarricense, así como el abordaje del sistema estructural y comunicativo del español. Por consiguiente, utilizar los contenidos de tales materias para organizar el currículo de inglés permitiría a los niños establecer conexiones significativas al abordar en inglés temáticas ya estudiadas en su lengua materna y que eran inherentes a su contexto cultural. La expectativa era que tal estrategia permitiera a estos estudiantes acelerar su adquisición de la segunda lengua y tener motivación para utilizar esta vía para comunicar sus ideas y sentimientos, así como para descifrar información impresa y auditiva en inglés.

En cuanto al enfoque pedagógico seleccionado para diseñar el currículum de inglés, inicialmente se utilizó el método audiolingüe. Según Larsen-Freeman, esta perspectiva educativa tiene como meta el uso comunicativo de la lengua extranjera, aprendiendo a utilizarla automáticamente sin detenerse a pensar. Los estudiantes logran lo anterior por medio de la formación de nuevos hábitos en el uso del inglés, superando las rutinas de uso de su idioma nativo ${ }^{20}$. En nuestro país este enfoque era novedoso en el decenio de $1970^{21}$ y fue seleccionado por las instructores del Plan Piloto precisamente porque

19 Villalobos Ellis, Entrevista, 12 de abril de 2011.

20 Diane Larsen-Freeman, Techniques and Principles in Language Teaching. (Oxford: Oxford UP, 1986) 31.

21 Lelia Villalobos, Entrevista abierta con Valenzuela y Álvarez. ELCL, Decanato FFL, Heredia, Costa Rica. 13 de mayo de 2009. 
ofrecía la posibilidad de formar individuos bilingües que pudieran no solo comprender sino producir en la lengua extranjera, siendo esta una de las metas fundamentales del proyecto.

Posteriormente, se termina de diseñar el currículo con base en el enfoque natural (Natural Approach). Krashen y Terrel ${ }^{22}$ enfatizan que los niños pueden adquirir una lengua extranjera de manera natural - tal y como se adquiere la lengua materna - lo que les permite comprenderla y utilizarla para fines comunicativos. Esta meta se alcanza exponiendo a los niños a situaciones cotidianas de la vida real, en las que el inglés se utilice como medio de comunicación. Según indica Villalobos Ellis ${ }^{23}$, esta estrategia condujo a las extensionistas, entre otras cosas, al diseño de materiales auténticos. Así, con base en las teorías del Enfoque Natural, se crearon diversos tipos de recursos para la enseñanza del inglés en primer ciclo.

La enseñanza-aprendizaje de las habilidades básicas del inglés (producción oral y escrita, comprensión auditiva y de lectura) se organizó bajo dos modalidades ${ }^{24}$. La primera se enfocó en desarrollar la habilidad de escucha y expresión oral entre los estudiantes del primer ciclo (primero, segundo y tercer grado). La segunda se concentró en potenciar en el alumnado de segundo ciclo (cuarto, quinto y sexto grado) las habilidades para la lectura y la expresión escrita. Las proyectistas justifican este procedimiento en el hecho que, en la realidad, los niños adquieren primero la habilidad de comprender lo que escuchan y luego desarrollan la expresión oral paulatinamente, y no es sino hasta una etapa posterior cuando los niños alcanzan la madurez [desarrollo motor y cognitivo] que les permite comprender textos y escribir ${ }^{25}$.

22 Stephen Krashen y Tracy Terrel, The Natural Approach: Language Acquisition in the Classroom (Hayward, CA: Alemany, 1983) 55-59.

23 Villalobos Ellis, Entrevista, 12 de abril de 2011.

24 Idem.

25 Sonya Vargas y Ruth Reuben, Plan Piloto de la Enseñanza del Inglés como Segunda Lengua: I Parte del Informe de 1979. Escuela de Literatura y Ciencias del Lenguaje, Universidad Nacional. 
El desarrollo del currículo de inglés —organizado en dos modalidades - se apoyó en unidades didácticas especialmente creadas para hacer frente a las necesidades de los estudiantes de la Escuela Joaquín Lizano. Indican Vargas, Reuben y Villalobos Ellis que este material formativo lo diseñaron con anterioridad a la implementación de los cursos de inglés en cada nivel escolar. Ello demandó múltiples y complejas tareas de investigación, pues todos los recursos didácticos serían inéditos. El proceso investigativo condujo a la creación de cincuenta y cuatro unidades didácticas; nueve para cada uno de los seis grados. Paralelamente a la creación de estos recursos y materiales, las extensionistas se vieron en la necesidad de procurar financiamiento externo para adquirir materiales tales como láminas de cartón, marcadores de distintos colores, pinceles de acuarela, y otros ${ }^{26}$. En 1979 el presupuesto para la compra de material debió realizarse externamente, ya que las gestiones realizadas internamente por las extensionistas para que la Universidad asumiera esos costos no fructificaron.

La producción de las unidades didácticas fue encargada al Departamento de Tecnología Educativa de la Universidad Nacional, pero por una u otra razón no cumplieron con ese compromiso ${ }^{27}$ y más bien postergaron hasta el último momento notificar a las extensionistas que no podían colaborar con el proyecto, lo que trajo como consecuencia que las clases de segundo grado se iniciaran hasta agosto, y no en marzo de 1981 como estaba programado. Esto también tuvo repercusiones directas en el desarrollo de las clases de inglés, ya que «algunas actividades tales como canciones y diálogos, contempladas en las unidades didácticas, no se pudieron realizar ${ }^{28}$. Por ello, una tarea adicional de las proyectistas fue la búsqueda de financiamiento externo, en vista de que la Universidad Nacional

26 Jorge Alfaro, Correspondencia interna (LI-0268-81) a Jorge Charpentier García, vicerrector de Extensión, Universidad Nacional. 26 de mayo de 1981.

27 Sonya Vargas, Ruth Reuben e Ileana Villalobos Ellis, Correspondencia interna a Jorge Alfaro Pérez, director Escuela de Literatura y Ciencias del Lenguaje. 29 de julio de 1981.

28 Desarrollo de Programa Académico: Plan Piloto de la Enseñanza del Inglés como Segunda Lengua. 1981. 
únicamente aportaba $1 / 4$ de tiempo para cada una de las extensionistas y no proveía presupuesto para el diseño y confección de materiales. Sin embargo, Reuben señala que «...por primera vez desde 1979 la Universidad Nacional nos ha autorizado $₫ 4.000 .00$ (cuatro mil colones) para la compra de material didáctico y nos envió rollos de película ${ }^{29}$. Pero este aporte no solventaba todos los gastos en los que debía incurrir el proyecto para desarrollar las clases con materiales auténticos. Con ello, la falta de financiamiento llevó al equipo del PPEIEP nuevamente a desplegar esfuerzos para captar recursos externos que, según Vargas, redundaron en que la Escuela Joaquín Lizano y los padres de familia de los niños participantes en el Plan Piloto hiciera un aporte de $ф 2.479 .00$ (dos mil cuatrocientos setenta y nueve colones ${ }^{30}$. Indica la profesora Vargas que debido a estas irregularidades, las clases de inglés en segundo grado no iniciaron hasta el lunes 3 de agosto de 1981, lo que retrasó el aprendizaje del inglés con metodología comunicativa por medio año ${ }^{31}$.

Esta constante limitación presupuestaria incidió a lo largo del desarrollo del proyecto en la cantidad de discentes a los que se les podía impartir las clases de inglés. En el cuadro 1, se muestra información que evidencia la variabilidad en el número de grupos por año con los que trabajó el PPEIEP a lo largo de su experiencia de extensión e investigación.

29 Ruth Reuben, Informe evaluativo. Primer Semestre 1983: Plan Piloto de la Enseñanza del Inglés como Segunda Lengua. 1983.

30 Vargas, Reuben y Villalobos Ellis, Correspondencia interna, 29 de julio de 1981.

31 Sonya Vargas, Ruth Reuben e Ileana Villalobos Ellis, Oficio a Jorge A. Alfaro Pérez, director Escuela de Literatura y Ciencias del Lenguaje. 24 de noviembre de 1981. 


\section{Cuadro 1. Grupos atendidos por el PPEIEP en la Escuela Joaquín Lizano (1979-1984)}

\begin{tabular}{|c|c|l|l|}
\hline Grado & $\begin{array}{c}\text { Año en que } \\
\text { el PPEIEP } \\
\text { impartió clase }\end{array}$ & $\begin{array}{l}\text { Número de grupos, cantidad } \\
\text { de estudiantes y horario }\end{array}$ & Tiempo de clase \\
\hline $1^{\circ}$ & $1979-1980$ & $\begin{array}{l}\text { 2 grupos de aprox. 25 estud.; } \\
\text { horario en la tarde }\end{array}$ & $\begin{array}{l}30 \text { minutos; } \\
4 \text { veces por semana }\end{array}$ \\
\hline $2^{\circ}$ & 1981 & $\begin{array}{l}\text { 2 grupos de aprox. 25 estud.; } \\
\text { horario en la tarde }\end{array}$ & $\begin{array}{l}30 \text { minutos; } \\
4 \text { veces por semana }\end{array}$ \\
\hline $3^{\circ}$ & 1982 & $\begin{array}{l}2 \text { grupos de aprox. 25 estud.; } \\
\text { de 7:40 a 8:20 a. m., } \\
\text { martes y jueves }\end{array}$ & $\begin{array}{l}80 \text { minutos; } \\
2 \text { veces por semana }\end{array}$ \\
\hline $4^{\circ}$ & 1983 & $\begin{array}{l}3 \text { grupos de aprox. 30 estud.; } \\
\text { de 7:40 a 8:20 a. m., } \\
\text { martes y jueves }\end{array}$ & $\begin{array}{l}80 \text { minutos; } \\
2 \text { veces por semana }\end{array}$ \\
\hline $5^{\circ}$ & 1984 & $\begin{array}{l}1 \text { grupo de aprox. 30 estud. } \\
80 \text { minutos; } \\
2 \text { veces por semana }\end{array}$ \\
\hline $6^{\circ}$ & 1985 & 1 grupo de aprox. 30 estud. & $\begin{array}{l}80 \text { minutos; } \\
2 \text { veces por semana }\end{array}$ \\
\hline
\end{tabular}

Fuente de información: Plan Piloto de la Enseñanza del Inglés como Segunda Lengua. I Parte del Informe de 1979 y Plan Piloto de la Enseñanza del Inglés como Segunda Lengua. Primer Semestre Informe Evaluativo 1983.

La información del cuadro 1 indica tanto momentos de crecimiento como de reducción en el número de grupos y de estudiantes involucrados. El proyecto inicia en 1979 con dos grupos de primer grado, cada uno con 25 estudiantes que reciben sus clases de inglés en la tarde. Al año siguiente (1981) se imparte clases a dos grupos de segundo grado; Vargas confirma que iniciaron el 3 de agosto y que «... cada docente tuvo a su cargo un grupo..., con horario de 30 minutos cuatro días a la semana» ${ }^{32}$. En 1982 se trabaja con terceros grados y se mantienen las condiciones del año anterior en cuanto al número de grupos y horario en que se imparten las clases. Para 1983 las extensionistas trabajan con tres grupos de cuarto grado; sin embargo se varía el 32 Sonya Vargas, correspondencia interna a Jorge Alfaro Pérez, director Escuela de Literatura y Ciencias del Lenguaje. 24 de noviembre de 1981. 
horario de las clases. Al respecto, Sonya Vargas indica que «...debido a que los niños son más receptivos a la adquisición de un segundo idioma durante las mañanas, decidimos ampliar el tiempo de cada lección y realizar nuestra labor siempre por la mañana $»^{33}$. De los tres grupos que se venían atendiendo, se reduce en 1984 a un solo grupo de quinto grado. Esta disminución se debió, según Ruth Reuben, al «...exceso de funciones que tenemos: Investigación, Extensión y Docencia ${ }^{34}$, por cuanto cada una de estas actividades demanda un arduo trabajo en el ámbito logístico, académico y de producción. En 1985 las condiciones de cuantía de grupos y horario de trabajo se mantienen; esto es, se imparten lecciones a un solo grupo en horario matutino.

\section{Currículo y principios comunicativos}

La base de lo logrado en los procesos de enseñanza y aprendizaje del PPEIEP radicó en el diseño del currículo delineado por las extensionistas, que muestra un esfuerzo innovador en tres aspectos fundamentales: 1 . no se apega a un texto educativo disponible en el mercado; 2. se fundamentó en unidades didácticas originales, diseñadas especialmente para cada nivel de la educación primaria; y 3. dichas unidades estaban fundamentadas en principios comunicativos para la enseñanza integral del inglés. La estrategia metodológica que utilizaron las extensionistas en la elaboración de las unidades didácticas consistió en diseñarlas en sintonía con temas abordados en el currículo de español, matemáticas, ciencias y estudios sociales. Además de lo anterior, y a fin de potenciar el desarrollo de la lengua extranjera en los aprendientes, el material se fundamenta en principios comunicativos tales como: aprendizaje basado en la experiencia; uso del inglés como único medio de comunicación durante la clase, aunado a la creación de un ambiente que estimule el uso del idioma

33 Sonya Vargas, Correspondencia interna dirigida al Lic. Jorge Alfaro Pérez, Director Escuela de Literatura y Ciencias del Lenguaje. 5 de marzo de 1982.

34 Reuben, Informe evaluativo, 1983. 
para la comunicación real; el estímulo a las inteligencias múltiples del discente, y el aprendizaje lúdico.

Se diseñaron nueve unidades para cada nivel de primaria, lo cual asciende a un total de 54 productos didácticos. Para el caso del primer grado, por ejemplo, Reuben ${ }^{35}$ indica que se escogieron y enseñaron las siguientes unidades con el vocabulario correspondiente:

- La familia (las personas más cercanas al niño)

- $\quad$ El aula de clase (objetos y personas)

- El alfabeto

- Los colores (seis)

- Las partes del cuerpo

- $\quad$ Los números (del 1 al 10)

- Los juguetes (algunos)

- Un paseo al campo

- La casa (aposentos)

- $\quad$ El supermercado (carnes, verduras, frutas, etc.)

- Animales domésticos

- Animales salvajes

Según Reuben:

se incluyeron [en las unidades didácticas] oraciones afirmativas, negativas, interrogativas e imperativas, todas en el tiempo presente. El material se enfocó en el sustantivo por el simple hecho de nombrar las cosas que rodean a los niños. Las estructuras verbales se subordinaron al proceso anterior pero se trató de utilizar principalmente estructuras en tiempos simples por considerar que los niños no dominan todavía estos aspectos gramaticales en su propia lengua.

35 Ruth Reuben y Sonya Vargas, I Parte del Informe de 1979. Plan Piloto de la Enseñanza del Inglés como Segunda Lengua. Escuela de Literatura y Ciencias del Lenguaje. 
La implementación del currículo de inglés en la Escuela Joaquín Lizano se llevó a cabo con éxito durante los seis años que duró el Plan Piloto. Vargas lo explica de la siguiente manera:

En secuencia alterna se enseñaron juegos y canciones que se relacionaban con los temas estudiados. También se crearon unas dramatizaciones de las cuales dos fueron presentadas en Asambleas de la Escuela de Literatura y Ciencias del Lenguaje. Una actividad relevante de la unidad didáctica Un Día de Campo contempló llevar a los niños al Club Campestre el Castillo ${ }^{36}$, con el fin de que escucharan y utilizan el inglés en un contexto real durante las actividades que se desarrollaron ${ }^{37}$.

El efecto de estas experiencias significativas en la población infantil se evidencia en el interés que despertaron por las lecciones de inglés entre los estudiantes y en la forma acelerada en que fueron adquiriendo la lengua extranjera. Reuben ${ }^{38}$ ilustra los resultados al indicar lo siguiente:

Los niños estaban encantados. Te voy a decir y tengo que ser realista, la clase que más esperaban era la clase de inglés...porque se cantaba, se bailaba, se jugaba, porque aprendían mucho inglés, porque había mucho movimiento corporal..., y los niños en esas etapas necesitan ese tipo de actividades: diversión, juegos, y canciones.

Vargas ${ }^{39}$ confirma esta sensación de los estudiantes de inglés de la Escuela Joaquín Lizano al recordar que «en cada clase, los chicos esperaban a las de inglés [las extensionistas] con gran entusiasmo, se pegaban a las ventanas para ver cuando llegábamos y saltaban

36 Centro de recreación familiar y social ubicado en la provincia de Heredia, Costa Rica.

37 Sonya Vargas y Ruth Reuben, Informe 1979 del Plan Piloto de la Enseñanza del Inglés como Segunda Lengua. 1979.

38 Ruth Reuben, Entrevista personal con Valenzuela. Sabana Norte, San José, Costa Rica. 2 de setiembre de 2011.

39 Sonya Vargas y Ruth Reuben, Entrevista, 30 de junio de 2014. 
de alegría. Esa era la mayor recompensa que podíamos recibir por el arduo trabajo de docencia e investigación que realizábamos».

Además, en un informe realizado por las extensionistas se indica que los diversos instrumentos de medición utilizados para valorar el aprendizaje de los estudiantes mostraron que «un $85 \%$ de los contenidos programáticos fueron asimilados por los mismos [aprendientes]. Estas cifras se desprenden de evaluaciones realizadas por cada una de las docentes». ${ }^{40} \mathrm{El}$ desempeño tan positivo de la población estudiantil tiene su cimiento en la estimulación que las proyectistas propiciaron para motivar y despertar el interés de los discentes por aprender la lengua. Vargas destaca que «los alumnos (...) mostraron gran entusiasmo y fascinación por el aprendizaje del inglés. Es muy grato poder decir que cinco meses después [de iniciadas las clases] los alumnos seguían manifestando el mismo interés y entusiasmo [por aprender la lengua] $\gg^{41}$.

Otro factor que confirma la satisfacción de los niños y padres de familia con respecto a la metodología comunicativa utilizada por las extensionistas es el hecho de que no hubo solicitud de padres para retirar a sus hijos del PPEIEP, ni tampoco de los mismos discentes, durante los seis años en que este operó en la Escuela Joaquín Lizano. Por el contrario, Vargas ${ }^{42}$ indica que los padres de los niños estaban tan complacidos con las dinámicas de enseñanza y aprendizaje implementadas por el PPEIEP, que llegaron incluso a brindar su aporte económico para colaborar económicamente para la compra del material didáctico que utilizaban sus hijos.

Además de potenciar en los pupilos el interés por aprender el inglés, las extensionistas del PPEIEP implementaron la estrategia de desarrollar las habilidades comunicativas del inglés por etapas. En el primer ciclo las proyectistas se enfocaron en apoyar a los estudiantes

\footnotetext{
40 Desarrollo de Programa Académico. Plan Piloto de la Enseñanza del Inglés como Segunda Lengua. 1981.

41 Vargas y Reuben, Informe 1979.

42 Vargas, Correspondencia interna, 24 de noviembre de 1981.
} 
en la adquisición de habilidades de expresión oral, comprensión auditiva y comprensión de lectura, y, no fue sino hasta el segundo ciclo que introdujeron la expresión escrita. Reuben y Vargas ${ }^{43}$ justifican esta estrategia aduciendo que los discentes de los primeros niveles de primaria apenas están desarrollando su habilidad de escribir en la lengua materna, por lo tanto consideraron contraproducente incorporar tempranamente la enseñanza de la escritura en inglés, ya que esto podría confundirlos.

\section{Autoformación y capacitación de proyectistas}

La formación de las académicas extensionistas del PPEIEP fue autodidacta desde el inicio mismo del proyecto, lo que les permitió generar nuevo conocimiento a partir de su proyección a la comunidad. De hecho, para la Universidad Nacional la extensión constituye:

...una tarea por medio de la cual la Universidad se interrelaciona con la comunidad nacional, de manera crítica y creadora. Por medio de ésta proyecta a la sociedad los productos del quehacer académico, lo que permite que se redimensione y enriquezca al percibir las necesidades de la sociedad, materializándose el vínculo universidadsociedad. Además, la extensión se justifica en la necesidad de nutrir la formación integral del docente, estudiante e investigador y fortalecer el compromiso que demanda el desarrollo humano ${ }^{44}$.

Con esta conceptualización, el extensionista es el docente que desarrolla capacidades de sensibilidad, pensamiento crítico, creatividad y humildad — entre otras - que le permiten compartir con grupos sociales que lo requieren el desarrollo teórico alcanzado a nivel universitario. Aplicados estos conocimientos en el mundo natural, los extensionistas logran calibrar ese conocimiento para que

43 Vargas y Reuben, Informe 1979.

44 Universidad Nacional, Dirección de Extensión. <http://www.extension.una.ac.cr/>; consultado el 19 de junio de 2012. 
responda de manera más pertinente a las necesidades particulares del contexto educativo y sociocultural del país, y, a menudo, este tratamiento resulta en la generación de nueva teoría.

Las extensionistas del PPEIEP logran así favoreser una mayor madurez profesional generada a partir de su participación en diversas tareas de docencia e investigación que asumen en el proyecto de extensión universitaria. Para mencionar solo una de ellas, se apunta a una de las dinámicas iniciales que debieron acometer y que consistió en buscar, analizar y seleccionar de manera crítica bibliografía disponible en el mercado para la enseñanza del inglés a niños, la que consistía de textos producidos en el extranjero para aprendientes de la lengua en el contexto de países extranjeros de habla inglesa. La evaluación del material permitió a las extensionistas percatarse que muchos de los contenidos temáticos y supuestos teóricos en que se fundamentan estas fuentes no se ajustaban a la realidad educativa costarricense, por lo cual seleccionaron metodologías, estrategias y contenidos académicos que consideraron pertinentes y eficientes en el contexto educativo del país. Procedieron entonces a adaptar los que requerían de ello, descartaron aquellos que consideraron irrelevantes o inviables para el ámbito formativo de la escuela primaria en nuestro país y a diseñar otros concordantes con la realidad del estudiante costarricense.

Las académicas continuaron evolucionando en sus habilidades como extensionistas mediante el aprendizaje que les proporcionaba la experiencia de la investigación-acción, llevada a cabo de manera paralela a la enseñanza del inglés a los niños. León y Montero definen la investigación-acción como «...el estudio de un contexto social donde mediante un proceso de investigación con pasos 'en espiral', se investiga al mismo tiempo en que se interviene» ${ }^{45}$. Este proceso lo llevaron a cabo las extensionistas a lo largo de seis años impartiendo clases de inglés en la Escuela Joaquín Lizano, tiempo durante el cual pilotearon

45 Citado en Hernández Sampieri y otros, 706. 
principios de enseñanza que habían modificado para satisfacer las necesidades de aprendizaje de la población infantil y depuraron los principios con fundamento en el aprendizaje obtenido de los resultados de la investigación que realizaban de manera simultánea.

Como se ha señalado anteriormente, las dinámicas del PPEIEP tenían como objetivo atender una necesidad de aprendizaje de idioma latente y apremiante de la época, lo cual es congruente con el impacto que se propone generar la investigación-acción, pues Sandín señala que en ésta se «...pretende propiciar el cambio social, transformar la realidad y que las personas tomen consciencia de su papel en ese proceso de transformación $»^{46}$. La tarea investigativa le permitió a las extensionistas maximizar sus habilidades de diseño de instrumentos de recolección de datos, sistematización de la información, construcción de conocimiento y generación de estrategias para mejorar los procesos de enseñanza y aprendizaje en el ámbito local, con miras a extender los resultados a nivel nacional.

Los procesos de autoaprendizaje señalados se enriquecieron también con los de capacitación formal que las mismas extensionistas se ocuparon de acceder por todos los medios que les fue posible. Por ejemplo, asistieron a un asesoramiento con la Dra. Marcella Frank, organizado por el Centro Cultural Costarricense Norteamericano en los meses de junio y julio de $1981^{47}$. Participaron en un seminario ofrecido por el Instituto de Tecnología Administrativa (ITEA), y en una conferencia en el Centro Cultural sobre «Técnicas para la Enseñanza del Inglés $»^{48}$. Además, concurrieron a tres seminarios que, según Reuben, se llevaron a cabo en 1983, y quien indica que:

...siempre con el interés de indagar acerca de nuevos métodos que enriquezcan el proyecto del Plan Piloto en la enseñanza del inglés a nivel de escuela primaria, hemos asistido a tres seminarios: Uno en

46 Citado en Hernández Sampieri y otros, 706.

47 Vargas, Correspondencia interna, 24 de noviembre de 1981.

48 Vargas, Correspondencia interna, 24 de noviembre de 1981. 
la Universidad de Costa Rica sobre Actualización Metodológica de la Enseñanza del Inglés, auspiciado por el Consejo Británico, con el Dr. Fernando Castaños. Otro en el Colegio María Auxiliadora sobre Enseñanza del Inglés a Niños en la Escuela Primaria, auspiciado por la Casa de Publicaciones Scott Foresman. Finalmente, asistimos a otro seminario en la Escuela de Literatura y Ciencias del Lenguaje, auspiciado por la misma con el nombre de «Adquisición de una Segunda Lengua», con el Dr. Bruce Fraser ${ }^{49}$.

Otras capacitaciones que permitieron a las extensionistas profundizar en sus conocimientos fueron por ejemplo, una charla con la Dra. Marta Montero, becada de la Organización de Estados Americanos (OEA), poseedora de un post doctorado de la Universidad de Harvard, quien trató el tema «La Concientización Cultural en la Enseñanza de un Segundo Idioma al Niño» ${ }^{50}$. Finalmente, las extensionistas también asistieron a un seminario en la ELCL, auspiciado por el Consejo Británico, y la UNAM (Universidad Nacional Autónoma de Méjico), sobre «Evaluación de Comprensión de Lectura de Textos en Inglés con Propósitos Específicos».

Estos y otros encuentros académicos en los que participaron las extensionistas les permitió no solo ampliar su conocimiento respecto a teorías y mejores prácticas en la enseñanza del inglés a niños desarrolladas e implementadas en el extranjero, sino verificar la validez de la teorización que ellas mismas estaban generando. Al respecto Reuben explica que:

Por invitación de la asesora de inglés Lic. Leonor Cabrera, por parte del Ministerio de Educación Pública, asistimos al Hotel Balmoral para exponer antecedentes, objetivos y el desarrollo de nuestro Plan Piloto ante representantes del Ministerio de Educación Pública, la Universidad de Costa Rica y algunos colegios bilingües del país. Como consecuencia de esta reunión, nos visitó la Prof. Jazmín

49 Reuben, Informe evaluativo, 1983.

50 Ídem. 
Calvo, quien labora en un centro educativo de enseñanza primaria en California, Estados Unidos. El objeto de su visita fue el de hacer un primer contacto con el área de inglés, con el fin de estudiar la posibilidad de un futuro intercambio de experiencias en cuanto a metodología, técnicas y otros aspectos del quehacer docente relacionado con niños y adolescentes. Este intercambio de experiencias se podría realizar por medio de talleres o seminarios dos veces al año, cuando esta profesora visita Costa Rica o por medio de cartas. El intercambio con personas o instituciones nacionales y extranjeras nos ha reafirmado la actualidad y eficacia de los métodos empleados [en el Proyecto Piloto implementado en la Escuela Joaquín Lizano] ${ }^{51}$.

\section{Efectos del PPEIEP}

El recuento histórico de las dinámicas del Plan Piloto permite establecer que lo actuado y los resultados de este proyecto constituyen la génesis a nivel país de la tropicalización de teorías foráneas de enseñanza y aprendizaje del inglés a niños. Sin embargo, las extensionistas no se limitaron a la adaptar materiales disponibles, sino que fueron innovadoras al derivar teorización propia mediante la investigación-acción. Ambas estrategias, permitieron que la pedagogía resultante respondiera de manera idónea a la realidad educativa costarricense y a las necesidades individuales de los estudiantes de la Escuela Joaquín Lizano. Por consiguiente, el PPEIEP posiciona a la UNA como institución de educación superior pionera en la promoción de la enseñanza y aprendizaje comunicativo del inglés a niños en Costa Rica. El impacto de la naturalización de las teorías extranjeras al ámbito nacional y de la generación de nuevo conocimiento en este ámbito específico de la enseñanza-aprendizaje de la lengua se hace evidente en el PPEIEP mediante el diseño e implementación de un currículo mayormente comunicativo para la enseñanza del inglés; la creación de material didáctico innovador; el desarrollo linguiístico

$51 \quad$ Reuben, Informe evaluativo 1983. 
que alcanzan un aproximado de cincuenta estudiantes de primaria; el impacto que el Plan Piloto generó para la posterior apertura en la ELCL de diversos proyectos de enseñanza de lenguas extranjeras dirigidos específicamente a la población infantil; y la incidencia que tuvo en la incorporación a nivel nacional del currículo de inglés en la escuela pública primaria, realizada por el MEP siete años después de finalizado el PPEIEP.

En relación con los principios comunicativos para la enseñanza del inglés a estudiantes de primaria, el PPEIEP sienta un precedente en cuanto a la adecuación e implementación de teorías generadas fuera del país, haciendo una selección de aquellas que eran pertinentes. Los principios fueron tomados de los métodos más recientes e innovadores de la época (finales de los setentas y principios de los ochentas), a saber, del Enfoque Natural y el Enfoque Comunicativo para la Enseñanza del Inglés. El hecho tiene gran relevancia porque con ello el equipo de extensionistas logró que los estudiantes seleccionados para participar en el PPEIEP pudieran utilizar el inglés efectivamente para comunicarse y no tan solo para leer y traducir, que era el objetivo que planteaban hasta ese momento los métodos tradicionales para la enseñanza de idiomas tales como el Método de Gramática y Traducción (Grammar Translation).

Además, las extensionistas generaron productos valiosos como La Guía del Maestro (para programas de los diferentes niveles escolares), un total de cincuenta y cuatro unidades didácticas (nueve por cada uno de los seis niveles en los que se impartió clases) y otros, que resultaron del esfuerzo de diseñar, pilotear y evaluar el material pedagógico auténtico creado para impartir lecciones a los estudiantes de la Escuela Joaquín Lizano. Como resultado de los procesos de depuración, este material resulta invaluable, pues los textos, ilustraciones, ejercicios, y recursos (canciones, poemas, etc.) eran significativos para los estudiantes nacionales, y se constituyeron en punto de referencia para otras investigaciones y proyectos en la enseñanza del inglés a niños. 
El efecto más destacado de toda iniciativa de enseñanza y aprendizaje será siempre la que deje huella en el ser humano, y, en este sentido, el proyecto logró instruir a un aproximado de 50 estudiantes de primaria en el idioma inglés. Al cabo de seis años, los discentes de la Escuela Joaquín Lizano involucrados en el PPEIEP desarrollaron habilidades para comunicarse en inglés a un nivel básico, pero comprensible. Este hecho es significativo puesto que la mayor parte de la población costarricense de la época no contaba con esta habilidad, ya que ninguna escuela primaria pública del país incluía el inglés en su currículo y solo un número muy reducido de instituciones educativas privadas impartía esta lengua extranjera a nivel de primaria.

Otro efecto en cuanto al de desarrollo humano se da en la formación de casi una decena de extensionistas, producto del relevo del equipo original del Plan Piloto. El PPEIEP desarrolló su labor de extensión con la participación de un equipo de trabajo que era reemplazado de manera intermitente en diferentes etapas de la ejecución del proyecto, en virtud de dos razones: 1) el alto volumen de trabajo que debían ejecutar las extensionistas con solo un cuarto de tiempo asignado para llevar a cabo de manera unísona tareas de docencia, investigación-acción y producción; y 2) la necesidad de la ELCL de contar con mayor número de profesionales con formación integral en estas áreas de desarrollo. En suma, entre 1979 y 1987 se prepararon un total de ocho extensionistas y su desarrollo profesional en extensión permeó el diseño e implementación de los diversos programas de formación de docentes en la enseñanza del inglés de la ELCL. Incluso existió la iniciativa de integrar al PPEIEP estudiantes de programas de formación de docentes de inglés de la ELCL, según se indica en el Lineamiento del Plan Académico 1983, en el cual el equipo de extensión establece como meta: «Informar a los docentes de nuestra Unidad Académica sobre los logros del proyecto...[y propone] involucrar a los estudiantes de la carrera en las 
actividades del proyecto» ${ }^{52}$. Esto da cuenta de la visión precursora de las extensionistas, quienes consideraron que el Plan Piloto podría ser de gran valía para el desarrollo profesional de los estudiantes de programas de formación de docentes de la ELCL, a la vez que el equipo de trabajo del proyecto se enriquecería con la ampliación de las diversas perspectivas y experiencias que pudiera aportar dicho recurso humano. Mediante las acciones antes señaladas, las extensionistas proporcionan conocimiento teórico más válido y ajustado a la realidad costarricense en los diversos programas de formación de docentes de inglés en los que trabajan a lo largo de su carrera profesional, con lo que cumplen uno de los objetivos centrales de la extensión universitaria, que según la Dirección de Extensión es: «contribuir a la creación y desarrollo de capacidades institucionales locales, a la transformación social, y a la recuperación y fortalecimiento del saber local, mediante la ejecución de programas, proyectos $\mathrm{y}$ actividades ${ }^{53}$.

El efecto de la investigación dejó su sello en la recolección y sistematización confiable de información, la depuración de prácticas pedagógicas, y la consolidación de habilidades cruciales de indagación educativa de las extensionistas. Al tiempo que se impartían las clases de inglés, las extensionistas diseñaban y aplicaban instrumentos para la recolección de datos que les permitiera evaluar la idoneidad de los principios de enseñanza y aprendizaje, además de los materiales didácticos diseñados para su práctica docente en la Escuela Joaquín Lizano. El resultado de la sistematización de datos les permitía ajustar dichos principios y materiales pedagógicos a las necesidades de los estudiantes y utilizar la versión depurada en el aula. Este proceso cíclico de prueba-depuración-prueba se repitió a lo largo de los seis años de operación del proyecto e hizo posible concluir

52 Lineamiento del Plan Académico 1983: Plan Piloto de la Enseñanza del Inglés como Segunda Lengua. 1983.

53 Universidad Nacional, Dirección de Extensión. Página Web http://www.una.ac.cr/index.php/areas/ extension; consultado el 16 de enero de 2014. 
con la generación de teorización propia, que se tradujo en unidades didácticas refinadas, programas de estudio, artículos y otros. No menos importante es el efecto de la investigación-acción en las propias extensionistas, ya que los procesos de este tipo de indagación fortalecieron su habilidad para recolectar información, analizar datos y experiencias, interpretar la información y generar conocimiento.

Por último, según se constata en el presente recuento histórico del PPEIEP, las extensionistas compartieron a lo largo del desarrollo del proyecto y al final del mismo (1978-1987) la teorización y productos emanados de la experiencia académica y de la investigación paralela que realizaban, socializándolos con diversas audiencias a nivel nacional y compartiéndolos formalmente con el MEP. Con ello aportaron a la integración paulatina del currículo de inglés en las escuelas públicas de primaria a nivel nacional, que realizara el MEP a mediados del decenio de 1990. Escalante ${ }^{54}$ indica que en 1994 la administración Figueres Olsen empieza la incorporación del inglés en la educación primaria del país, mediante un proyecto piloto del MEP que incluyó una muestra de escuelas del país, teniendo como meta abarcar progresivamente la mayor parte de los centros educativos de primaria de Costa Rica.

\section{Tercera fase del PPEIEP. Evaluación y reestructuración (1985- 1987)}

A finales de 1985 surgió la necesidad de evaluar el PPEIEP con el fin de derivar el aprendizaje global resultante de dicha experiencia. El Dr. Carlos Francisco Monge Meza, director de la ELCL en esa época solicita a la coordinadora del proyecto piloto lo siguiente:

54 Manuel Escalante, «The History of Education in Costa Rica». Ponencia presentada en el V Congress of the Americas Popular Culture Association Meeting, Universidad de las Américas. (Puebla, México: University of La Verne, California. Junio 2001). 
Con el propósito de elaborar una recomendación ante la Asamblea de Escuela, hemos estimado indispensable que las personas que han integrado a través de todos estos años (1979 hasta 1985) presenten un informe general por año, de las actividades y situaciones más sobresalientes y significativas acontecidas desde entonces ${ }^{55}$.

El equipo de trabajo del PPEIEP se avoca a cumplir con lo dispuesto por el señor director generando un informe final, que se sustentaría en las evaluaciones y reportes anuales que se habían realizado sobre la marcha del proyecto. Por ejemplo, en el informe anual de 1983 Reuben indica que luego de cinco años de vigencia del Plan Piloto, la evaluación demostró que los niños participantes del proyecto habían desarrollado habilidades comunicativas del inglés que sorprendieron a los propios padres de familia, quienes agradecieron el progreso singular del que eran parte sus hijos en uso del inglés con fines comunicativos ${ }^{56}$.

Además, en esta etapa de evaluación del proyecto se reestructuraron algunos componentes en el campo humano, lo cual permitió que la evaluación tuviese aún mayor validez al realizarse el proceso desde la perspectiva múltiple de integrantes extensionistas con experiencia y otras novatas. Por ejemplo, L. Villalobos indica que algunos miembros del equipo de trabajo que inició en 1985 dejan el proyecto y se incorpora a nuevas extensionistas. Las académicas Agnes Flores Gámez, Lelia Villalobos Rodríguez, Ileana Saborío Pérez y Ruth Reuben Coronado conformaron el grupo de esta segunda etapa. Sin embargo, solo Saborío y Reuben concluyeron el proyecto en 1986, siendo la coordinadora del programa Ruth Reuben ${ }^{57}$. Este dúo heterogéneo - por ser Saborío extensionista con menos experiencia, unida a Reuben que la tenía amplia - concluyó las tareas de valoración del PPEIEP con gran propiedad.

\footnotetext{
55 Carlos Francisco Monge, Oficio LI-0206-85. ELCL, UNA, 30 de julio de 1985.

56 Reuben, Informe evaluativo, 1983.

57 Lelia Villalobos, Entrevista abierta con Valenzuela y Álvarez. ELCL, Decanato FFL, Heredia, Costa Rica. 13 de mayo de 2009.
} 
Otro aspecto relevante en la tercera etapa del proyecto, la de evaluación, señala Villalobos ${ }^{58}$, es lo que se concluye respecto al ámbito pedagógico. Se devela la pertinencia y éxito de la integración de principios comunicativos utilizados para la enseñanza del inglés en la Escuela Joaquín Lizano, en reemplazo de algunos principios de enseñanza más tradicionales con los que se operó al inicio del proyecto. También se comprueba la premisa de las extensionistas en cuanto a que las habilidades del lenguaje que se debían enfatizar en el primer ciclo eran la comprensión auditiva y la producción oral, con el fin de que las otras habilidades no interfirieran con el proceso de aprendizaje de la lecto-escritura del español, pero que en el segundo ciclo todas las habilidades del inglés se debían incluir en el proceso de aprendizaje con igual intensidad, en vista de que en estos niveles los estudiantes ya han superado la fase crítica del proceso de aprendizaje de lectura y escritura de la lengua materna.

Producto de la experiencia recogida a lo largo de las dos fases de la puesta en práctica del PPEIEP (1979-1982 y 1983-1985) y la de evaluación (1986-1987), la Villalobos Ellis ${ }^{59}$ ratifica que en 1986 se presentó al MEP una propuesta pedagógica para la enseñanza del inglés en todos los centros educativos de primaria del país, pero el MEP nunca respondió a esa petitoria. Aun así, tanto la experiencia de investigación-acción como los productos humanos y académicos resultantes generaron un cambio de paradigmas en la enseñanza del inglés a niños, que inicialmente favoreció solo a los estudiantes de la Escuela Joaquín Lizano, pero que con el paso del tiempo permeó en los planes de formación de docentes de inglés en la ELCL, con lo cual se ha impactado por décadas a generaciones de estudiantes de primaria. Además, el conocimiento generado durante el desarrollo del proyecto ha sido punto de partida de otros proyectos de investigación y extensión de la ELCL. 


\section{Conclusiones}

Las extensionistas del PPEIEP son pioneras de la enseñanza del inglés a la población infantil en Costa Rica y un equipo académico innovador, audaz y adelantado a su tiempo. Ha sido la investigación-acción el mecanismo de indagación utilizado por estas extensionistas, método que solo recientemente se ha empezado a incorporar paulatinamente en proyectos de investigación cuyo objeto de estudio es la enseñanza del inglés a niños, en las universidades públicas del país. Este hecho marca el liderazgo de la ELCL en este campo, ya que no solo estudia los fenómenos de la enseñanza del inglés, sino que diseña propuestas de mejoramiento y las ejecuta, permitiendo este proceso cíclico depurar los programas de formación docente de la misma unidad académica.

Las extensionistas también fueron audaces al utilizar principios comunicativos para la enseñanza del inglés a niños creados por expertos internacionales en el decenio de 1970. El avanzado desarrollo de habilidades críticas de las extensionistas fue el factor que les permitió implementar una herramienta pedagógica de la cual no tenían la seguridad de que fuese efectiva. Fue tan osada esta iniciativa en aquel momento que no es sino hasta 2012 que el MEP empieza a impulsar el principio de aprendizaje lúdico y el aprendizaje mediante la experiencia como mecanismos eficientes para la enseñanza-aprendizaje significativo de los estudiantes, lo que se evidencia en reportajes del periódico La Nación como el que se titula «La resistenciadocente $»^{60}$.

La innovación de las extensionistas también deja su huella en la producción de programas de enseñanza de inglés para pri- maria y de unidades didácticas basadas en principios comunica- tivos, incluyendo en ellas material didáctico original y pertinente al ámbito educativo costarricense. Estos productos constituyen la primera bibliografía nacional especializada en la enseñanza del

60 José Ramiro Rodríguez, La Nación Digital. Opinión. La resistencia docente. <http://www.nacion. com/opinion/foros/resistencia-docente_0_1409859005.html>; consultado el 12 de junio de 2014. 
inglés a población infantil, dando esto el impulso a otros educadores para teorizar y diseñar productos acorde con las necesida- des de los estudiantes del país.

Las pioneras demostraron un nivel tan alto de compromiso y solidaridad que fueron ejes fundamentales para que el equipo de trabajo se renovara constantemente con el ingreso de nuevas colegas, quienes le aportaron al proyecto diversas visiones, a la vez que se nutrían de su experiencia de investigación-acción, esencial en la formación de ocho extensionistas. Este personal con visión más integral entre la teoría y la práctica en el ámbito universitario y comunitario se constituye en el puente que retroalimenta la práctica universitaria con la realidad del mundo natural.

En 1995, siete años después de finalizado el PPEIEP, algunas de sus extensionistas formulan un nuevo proyecto para la enseñanza del inglés a niños denominado Programa de Capacitación para Profesores de Inglés (PROCAPRI), proyecto de extensión e investigación de la ELCL. En 2014 este proyecto cumple 19 años de vigencia y ha capacitado con principios comunicativos a 540 docentes del área central y rural del país, beneficiando a un promedio de 600.000 estudiantes de inglés de primaria. El quehacer y la innovación del PROCAPRI se retomará en otros artículos, pero sirva la mención para resaltarlo como producto derivado de las acciones y resultados obtenidos por el equipo pionero de investigación del PPEIEP. 